\title{
Fluorine F 18 FDHT
}

National Cancer Institute

\section{Source}

National Cancer Institute. Fluorine F18 FDHT. NCl Thesaurus. Code C112496.

A radioconjug ate containing a derivative of the androgen testosterone, 16-beta-fluoro-5alpha-dihydrotestosterone (FDHT), labeled with the radioisotope fluorine F18 (18F-

FDHT), with potential use as an imaging agent for positron emission tomography (PET).

Upon administration, 18F-FDHT binds to the androgen receptor (AR). Upon PET imaging, AR-expressing prostate tumor cells can be imaged and assessed. 